\title{
Fabrication Trials of Round Strands Composed of Coated Conductor Tapes
}

\author{
D. Uglietti ${ }^{1}$, R. Wesche ${ }^{1}$, and P. Bruzzone ${ }^{1}$
}

\begin{abstract}
Various techniques have been proposed in order to assemble coated conductors tapes into high current strands. In this work the preparation of high current capacity round strands starting from industrial coated conductors has been investigated. Round, twisted strands (about $50 \mathrm{~cm}$ long) consisting of stacked tapes sandwiched between two semi-circular copper profiles have been assembled following two manufacturing routes: in one case the stack was first soldered to the copper profiles and than twisted; in the second method a twisted strand (stacked tapes between copper profiles) was soldered. The round conductors carry over $400 \mathrm{~A}$ at $77 \mathrm{~K}$ in self field. Three types of solder alloy were tested: BiSn, InSn and PbSn. The critical current variation under bending strain was also measured: critical current retention of $99 \%$ was observed up to $0.3 \%$ peak bending strain. At $0.6 \%$ bending strain the strands prepared with $\mathrm{PbSn}$ solder exhibit a reversible reduction of the critical current of less than $\mathbf{2 \%}$, irrespective of the manufacturing route. Such round strands could be used to manufacture flat cables with conventional cabling methods.
\end{abstract}

Index Terms - Coated conductor, HTS cable, round strand.

\section{INTRODUCTION}

$\mathrm{L}$ ARGE magnets (for example fusion magnets and detector

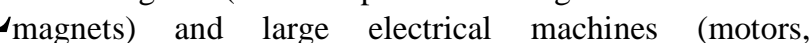
transformers, etc...) should be constructed using cables able to carry from few kA up to hundred kA of current. Technology for building such cables (cable in conduit, Rutherford cables, flat cables) has been established since several decades for $\mathrm{NbTi}$ and $\mathrm{Nb}_{3} \mathrm{Sn}$ wires, which are classical Low Temperature Superconducting (LTS) materials, and for Bi2212 wires, which is a High Temperature Superconducting (HTS) material; all these materials are available as round wires from industrial manufacturers. Technology for building cables composed of tapes, like Bi2223 tapes and coated conductor tapes, is still in the early development stage. Up to now, the following designs have been proposed and tested on short length (few meters): stacked tape in copper [1], Roebel cable [2], and flexible cable [3]. An alternative way [4] of building a large magnet with HTS tapes is to split the winding pack in blocks (consisting of stacked tapes), which are jointed together. Nexans [5] has developed a technique to fabricate round hollow wires (about $2 \mathrm{~mm}$ diameter) using coated

Manuscript received October 9, 2012. This work was supported in part by EFDA work program WP12-DAS-01-T08.

D. Uglietti, R. Wesche and P. Bruzzone are with EPFL-CRPP, Fusion Technology, CH-5232 Villigen PSI (phone: +41 56310 5820; fax: +41 56 3103729; e-mail: davide.uglietti@psi.ch) conductor tapes.

In this paper the objective is to develop round strands using coated conductors, with a particular focus on magnets for fusion applications; that means that the overall current density can be as low as $100 \mathrm{~A} / \mathrm{mm}^{2}$, and bending radius of the final cable larger than one meter. The really important features a cable for fusion magnets should have are easy, reliable and cheap manufacturing process for length of at least one kilometer. Bi2223 tapes and coated conductor tapes are presently rather expensive, nevertheless it is still important to develop a method for assembling cable from tapes, because one may speculate that such expertise may be applied in the future to new technical superconducting materials, (i.e. picnitides) which could be available from industrial production only as tapes.

In this work a variation of the method described in [1] is studied: here, a stack of tapes is sandwiched between two copper profiles and then soldered, resulting in a solid, round conductor (strand), similar to classical LTS strands. Two fabrication routes have been investigated, the main concern being whether coated conductors can withstand the mechanical forces (in particular shearing forces) during the twisting process. The evolution of the critical current under bending strain was also measured.

\section{STRAND MANUFACTURING}

Copper electroplated coated conductors from Superpower (SCS3050) were chosen for the fabrication of the strands. $\mathrm{Cu}$ coating makes the soldering process much easier than for an Ag coated tape. The tape is $3 \mathrm{~mm}$ wide; the critical current $\left(I_{c}\right)$ measured every 5 meters is between $79 \mathrm{~A}$ and $82 \mathrm{~A}$ and the exponential $n$ value between 24 and 28. For each sample, eight tapes have been cut and pre-soldered, they were stacked and then sandwiched (with the ceramic layer on the same side for all the tapes) between two copper semi-circular profiles which are $50 \mathrm{~cm}$ long. Photographs of the assembled strand and after soldering are presented in Fig.1. The copper profiles were obtained by machining a $3 \mathrm{~mm} \emptyset$ copper bar; long length can
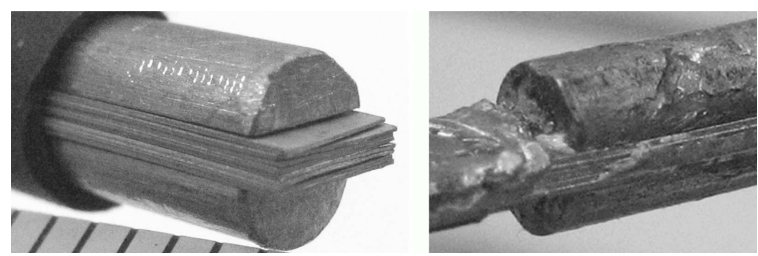

Fig. 1. Left: photograph of the assembled strand before soldering and twisting; the thermo-retractable tube is also visible. Division is $1 \mathrm{~mm}$. Right: after soldering. 
be easily obtained from industrial production by drawing. Few short pieces ( 1 to $2 \mathrm{~cm}$ long) of plastic retractable tubes were used to hold copper profiles and the stacked tapes together. In order to manufacture a fully soldered and twisted strand, two fabrication routes could be chosen: in one the assembled strand is soldered before being twisted (samples labeled ST, i.e. "Solder and Twist"); a second option is first to twist the assembled strand and then solder all the components (sample labeled TS, i.e. "Twist and Solder").

The strands were soldered with a hand-held soldering iron in a continuous process: the solder alloy is melted on the strand over few $\mathrm{cm}$, clamped with few clamps, then moving forward and repeat the process. The choice of the soldering alloy was mainly driven by the melting temperature: in fact, the critical current of coated conductors is degraded after exposure to temperatures exceeding $250^{\circ} \mathrm{C}$ for tens of seconds. Therefore three low temperature alloys were selected for the trial fabrications: 50wt.\%In50wt.\%Sn (InSn), 50wt.\%Bi50wt.\%Sn (BiSn) and 40wt.\%Pb60wt.\%Sn (PbSn). Zinc chloride based flux was used with InSn and BiSn solder, while $\mathrm{PbSn}$ is a standard flux cored wire.

The twisting process for the strands presented here was the following: the strand is hold between two rotating late chucks, $50 \mathrm{~mm}$ apart: one chuck is kept fixed while the other is rotated of $45^{\circ}$; when the clamp is released the strand springs back, but some twist (about $20^{\circ}$ ) is maintained. The process is carried out over the whole length of the strand and repeated for few times, till the desired twist pitch is obtained. Such technique ensures a uniform twist pitch (within 2\%) over the whole length The length of the twist pitch depends on the application, and it is usually calculated taking into consideration AC losses, operating conditions, etc... No specific design for future fusion magnets is yet available, thus twist pitch values between 280 and $360 \mathrm{~mm}$ were chosen in order to have a comfortable margin with respect to the critical value of about $200 \mathrm{~mm}$, which was measured on a stack of four tapes [6]. The maximum strain experienced by the tapes in the twisted strand is estimated to be less than $0.05 \%$ (formula from [1], pag.3, was used).

The current terminals at the strand ends were prepared by soldering a $2 \mathrm{~mm}$ thick copper plate $(20 \mathrm{~mm}$ wide and $80 \mathrm{~mm}$ long) to the tapes, which were cut several centimeters longer than the copper profiles. Copper cables were bolted to the copper plates.

\section{MEASUREMENTS AND DISCUSSION}

The critical current $(1 \mu \mathrm{V} / \mathrm{cm}$ criterion) of the strands was measured at $77 \mathrm{~K}$ in liquid nitrogen; $n$ values were calculated between $1 \mu \mathrm{V} / \mathrm{cm}$ and $5 \mu \mathrm{V} / \mathrm{cm}$. Six voltage taps were soldered along the strand and the total distance between the outermost voltage taps is $35 \mathrm{~cm}$. The nominal $I_{c}$ is $80 \mathrm{x} 8=640$ A at $77 \mathrm{~K}$, but, because of the self field, the actual critical current is estimated to be between $415 \mathrm{~A}$ and $450 \mathrm{~A}$.

In order to measure the critical current evolution under bending strain, the strands were bent around a wooden support at room temperature. Then the whole assembly was immersed in liquid nitrogen. Several supports with bending radius ranging from $1.5 \mathrm{~m}(0.10 \%$ peak bending strain $)$ to $0.25 \mathrm{~m}$ $(0.60 \%$ peak bending strain) were prepared.

\section{A. "Solder and Twist" strands}

The ST1 strand was prepared with BiSn, which is a rather brittle alloy due to the presence of $\mathrm{Bi}$. The critical current of the straight strand was 445 A $(n=27)$, which lies in the expected range. During the twisting process cracks appeared in the solder between the tapes and between the stack and the copper profiles; cracks were repaired using $\mathrm{InSn}$ alloy. $\mathrm{I}_{\mathrm{c}}$ decreased to 342 (see Fig. 2) after the twisting process (final twist pitch was $360 \mathrm{~mm}$ ). A decrease of $I_{c}$ is expected, due to the increase of the component of the self field perpendicular to the wide face of the tape: such component is proportionally increased when the twist pitch is reduced. Nevertheless, such large degradation seems to be excessive. Indeed the E-I curves (see Fig. 3) show a linear increase of the electric field at currents much lower than $I_{c}$, which is usually considered a signature of fracture in the superconducting layer. Crack formation and strong $I_{c}$ degradation continued also for relatively small values of bending strain; the test was stopped at $0.3 \%$ bending strain.

In contrast with strand ST1, strand ST2 was soldered with InSn solder alloy, which is much more ductile than BiSn. The critical current of the soldered, non twisted strand is $438 \mathrm{~A}$, in agreement with the expected value. During the twisting

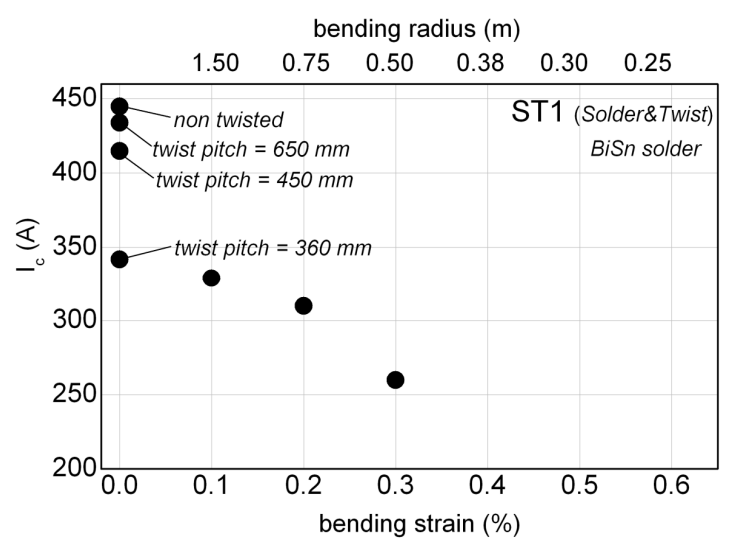

Fig. 2. Critical current versus bending strain for sample ST1. The corresponding bending radius is indicated on the upper axis. The values at $0 \%$ bending strain refer to $I_{c}$ measured during the twisting process.

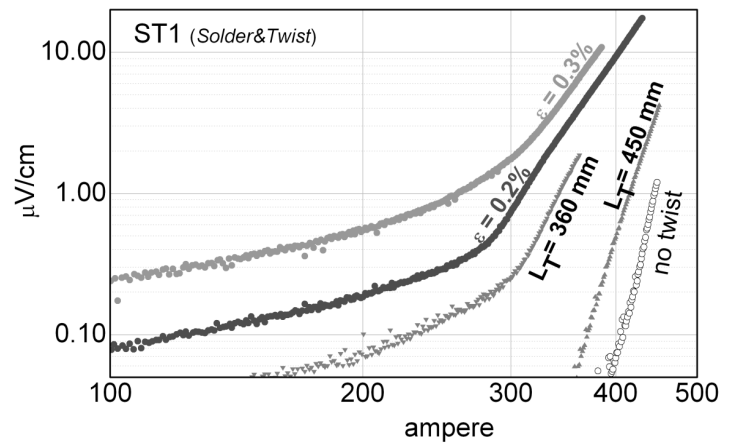

Fig. 3. Electric field versus current measured at $0 \%$ bending strain (no twist, $450 \mathrm{~mm}$ twist pitch and $360 \mathrm{~mm}$ twist pitch), $0.2 \%$ and $0.3 \%$ bending strain for sample ST1. 
process cracks were not observed and the final twist pitch was set at $300 \mathrm{~mm}$. Critical current reduction after the twisting process was about $6 \%$ (see Fig. 4), much smaller the one measured on ST1 (it was 23\%); in addition the E-I curves were simply shifted to lower currents, and the $n$ value was only slightly reduced from 22 to 20 . During bending, negligible degradation was observed up to $0.30 \%$ bending strain. From $0.30 \%$ bending strain, cracks appeared after each bending step and $I_{c}$ decreased; after each bending step the damaged spots were repaired by soldering locally with SnIn. The final $I_{c}$ at $0.60 \%$ bending strain was $385 \mathrm{~A}$; after the measurement at $0.6 \%$ bending strain, a plastic deformation of the strand was observed, corresponding to a bending radius of about $0.75 \mathrm{~m}(0.25 \%$ bending strain). The critical current was measured again, and the value was found to be the same as the value measured at $0.6 \%$ bending strain, indicating that the degradation of $I_{c}$ is irreversible. This is supported also by the analysis of the E-I curves, which show linear slope when the bending strain was exceeding $0.3 \%$.

Strand ST3 was soldered with PbSn; the $I_{c}$ values (see Fig. 4) before and after twisting are comparable to the ones measured in ST2; $n$ values were 24 and 20 respectively. During bending no de-lamination was observed and $I_{c}$ reduction was smaller than in ST2, with only $2 \%$ reduction at $0.6 \%$ bending strain. In contrast with ST1 and ST2, in ST3 the E-I curves at $0.6 \%$ bending strain are little changed with respect to the ones at $0 \%$, indicating that no cracks are present in the superconducting layer. Indeed, after bending at $0.6 \%$ the strand was straightened, and the critical current recovered the initial value.

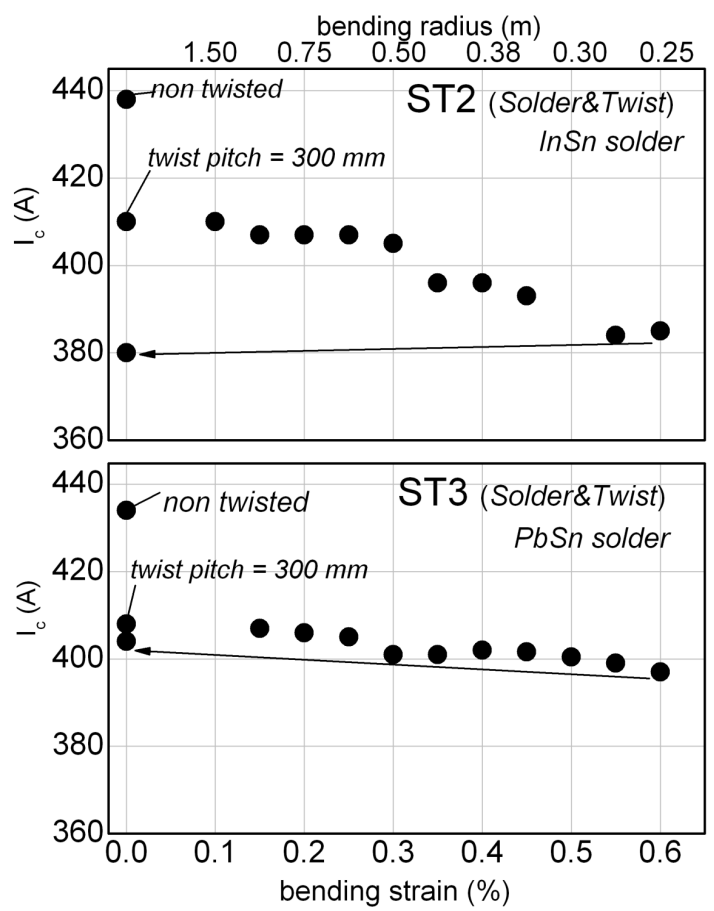

Fig. 4. Top: critical current versus bending strain for sample ST2. Bottom: critical current versus bending strain for sample ST3. The corresponding bending radius is indicated on the upper axis.

\section{B. "Twist and Solder" strands}

In these samples the twisting process was carried out before soldering the strands. Strand TS1 was prepared at the same time with ST2, and it was soldered with InSn. The critical current of the straight, twisted strand is 405 A (see Fig. 5), a value comparable to the one measured in ST2 and ST3 after the twisting process. No reduction was observed up to $0.40 \%$ bending strain, at $0.45 \%$ bending strain $I_{c}$ decreased to $385 \mathrm{~A}$ (about 5\% reduction) and remained constant till $0.60 \%$ bending strain. After straightening the strand, the critical current did not recover the initial value. As observed in the previous experiments, electric field at low current appears when irreversible degradation of $I_{c}$ takes place.

Sample TS2 was prepared like TS1, but PbSn was used for soldering. The critical current of the straight, twisted strand is 415 A (see Fig. 4b), in line with the expectations. The evolution of the critical current as a function of bending strain was similar to the one observed in sample ST3, i.e. a little reduction of $I_{c}$ which recovered to the initial value once the strand is straightened. In Fig. 6 the reduced critical current (i.e. $I_{c}$ divided by $I_{c}$ of the twisted strand at $0 \%$ bending strain) is plotted for all the sample apart from sample ST1 (not shown in Fig. 5a,b), whose solder alloy was too brittle. All other samples have a $99 \%$ critical current retention up to at least $0.3 \%$ bending strain. At $0.6 \%$ bending strain the samples soldered with InSn had an irreversible reduction of $I_{c}$ of about $6 \%$ to $7 \%$ (see fig.5), while the samples soldered with $\mathrm{PbSn}$ had a reversible reduction limited to $2 \%$. Therefore the $\mathrm{PbSn}$ alloy appears to be the best choice for soldering this kind of strands.

During bending the strand were arranged on the wooden support so that in two sections of the strand the tapes were

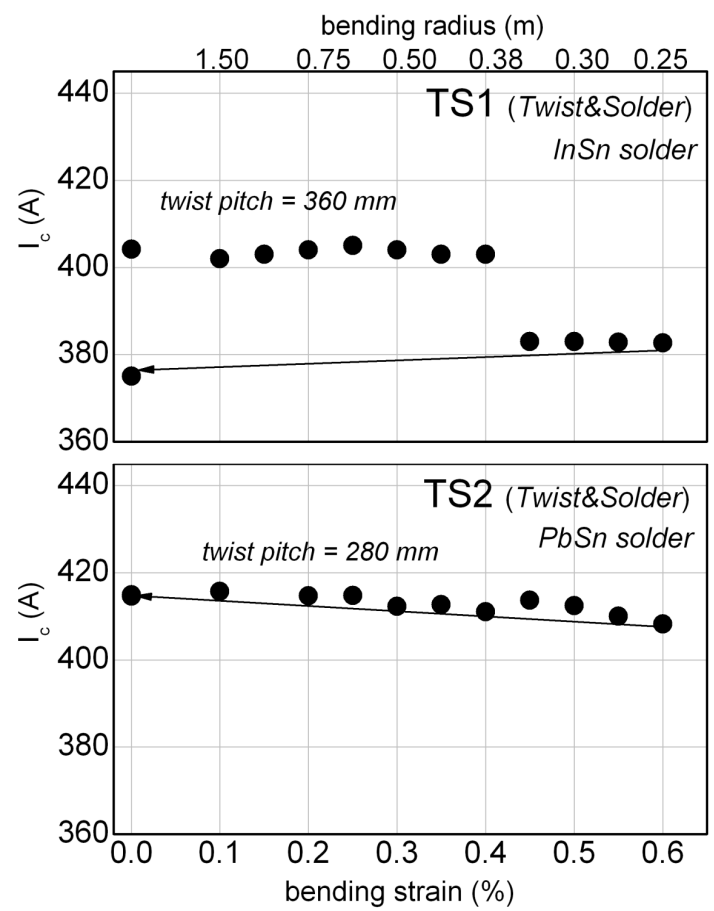

Fig. 5. Top: critical current versus bending strain for sample TS1 Bottom: critical current versus bending strain for sample TS2. The corresponding bending radius is indicated on the upper axis. 
subjected to in-plane bending. It as been shown [7] that $I_{c}$ of 4 $\mathrm{mm}$ wide coated conductors under in-plane bending is reversible (less than 5\% reduction) down to bending radius of at least $0.3 \mathrm{~m}$. The measurements on samples ST3 and TS2 are in agreement with the ones on single tapes. In samples (ST2 and TS1) which exhibited irreversible degradation of $I_{c}$, it is supposed that, when the tapes and the copper profiles detach from each other, the strain could locally assume high values (often referred to as $\mathrm{Z}$ bending) exceeding the irreversible tensile strain limit (about $0.6 \%$ ).

No significant difference was observed between the two fabrication routes, as shown in Fig. 6, although one may expect that the "Twist and Solder" route is preferable because the tapes in the strand will be in strain free state after the fabrication process, while some strain is present in the tapes if the strand is first solder and then twisted. Apart from sample prepared with BiSn solder, no degradation of $I_{c}$ was observed during the twisting process of a soldered strand. The characteristics of the more relevant strands (ST2, ST3, TS1 and TS2) are summarized in Table I.

Strands like the ones described here are expected to carry over $1000 \mathrm{~A}$ at $4.2 \mathrm{~K}$, in background field of $15 \mathrm{~T}$. Such type of strands could be assembled to form a flat cable. In the case an eight strands flat cable whose twist pitch is $1000 \mathrm{~mm}$ is assembled, the bending strain experienced by each strand at the cable edge can be estimated to be about $0.1 \%$. Therefore the bending strain margin before reaching $0.6 \%$ strain would be $0.5 \%$, corresponding to a bending radius of $0.3 \mathrm{~m}$. Such flat cable is expected to carry about $12 \mathrm{kA}$ at $4.2 \mathrm{~K}$, in background

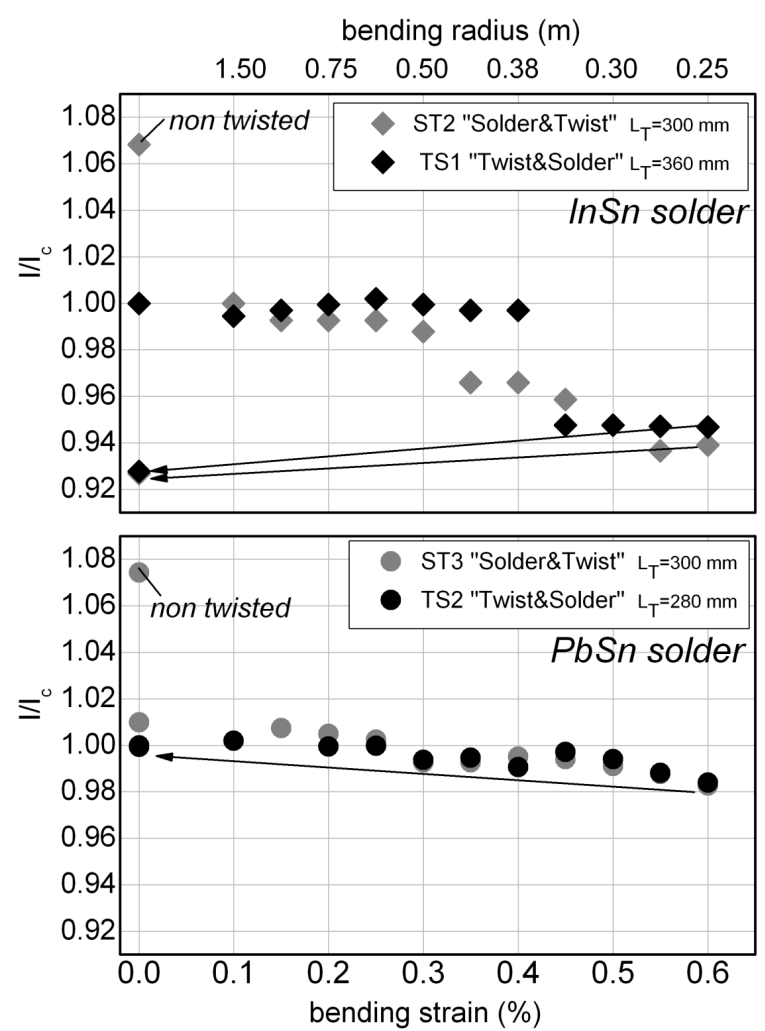

Fig. 6. Top: reduced critical current as a function of bending strain for samples soldered with InSn, Bottom: reduced critical current as a function of bending strain for samples soldered with $\mathrm{PbSn}$. field of $15 \mathrm{~T}$.

TABLE I

SAMPLE CHARACTERISTICS

\begin{tabular}{lccccc}
\hline \hline & $\begin{array}{c}\text { Solder } \\
\text { alloy }\end{array}$ & $\begin{array}{c}\text { Twist } \\
\text { pitch }\end{array}$ & $\begin{array}{c}\mathrm{I}_{\mathrm{c}} \text {, non } \\
\text { twisted }\end{array}$ & $\begin{array}{c}\mathrm{I}_{\mathrm{c}}, \\
\text { twisted }\end{array}$ & $\begin{array}{c}\mathrm{I}_{\mathrm{c}} \text { reduction at } \\
0.6 \% \text { bending }\end{array}$ \\
\hline ST2 & $\mathrm{InSn}$ & $300 \mathrm{~mm}$ & $438 \mathrm{~A}$ & $410 \mathrm{~A}$ & $6 \%$ \\
ST3 & $\mathrm{PbSn}$ & $300 \mathrm{~mm}$ & $434 \mathrm{~A}$ & $404 \mathrm{~A}$ & $2 \%$ \\
TS1 & $\mathrm{InSn}$ & $360 \mathrm{~mm}$ & - & $404 \mathrm{~A}$ & $5 \%$ \\
TS2 & $\mathrm{PbSn}$ & $280 \mathrm{~mm}$ & & $415 \mathrm{~A}$ & $2 \%$ \\
\hline \hline
\end{tabular}

\section{SUMMARY}

Twisted round strands $\left(3 \mathrm{~mm} \emptyset, I_{c}>400 \mathrm{~A}\right.$ at $77 \mathrm{~K}$ in selffield) composed of stacked coated conductor tapes sandwiched between two copper profiles have been fabricated. Various types of solder alloy and two fabrication routes have been attempted. Both routes (Solder\&Twist, Twist\&Solder) provided similar results; the choice could be done by considering which one is easier to apply in industrial production. All the samples soldered with $\mathrm{InSn}$ and $\mathrm{PbSn}$ solders conserved the initial value of the critical current up to $0.3 \%$ bending strain. At larger bending strain, the samples soldered with InSn had an irreversible reduction of $I_{c}$ as large as $7 \%$, while the samples soldered with $\mathrm{PbSn}$ had a reversible reduction of $I_{c}$ as little as $2 \%$.

The type of strand studied in this paper could carry over $1000 \mathrm{~A}$ at $4 \mathrm{~K}, 15 \mathrm{~T}$ background field; a flat cable composed of such strands could carry about $12 \mathrm{kA}$ at $4 \mathrm{~K}$ in $15 \mathrm{~T}$ background field and could be bent over $0.5 \mathrm{~m}$ bending radius (the reversible degradation of $I_{c}$ would be limited to $2 \%$ ). The construction of longer strands and a simple cable are under way.

\section{ACKNOWLEDGMENT}

The authors thank the Paul Scherrer Institute (PSI) for the technical support.

\section{REFERENCES}

[1] M. Takayasu, L. Chiesa, L. Bromberg and J. V. Minervini, "HTS twisted stacked-tape cable conductor", Superconductor Science and Technology, vol. 25, 2012, 014011 .

[2] S. I. Schlachter, W. Goldacker, F. Grilli, R. Heller, and A. Kudymow, "Coated Conductor Rutherford Cables (CCRC) for High-Current Applications: Concept and Properties", IEEE Trans. Appl. Supercond., vol. 21, 2011, 3021-4.

[3] D. C. van der Laan, X. F. Lu, and L. F. Goodrich, "Compact $\mathrm{GdBa} 2 \mathrm{Cu} 3 \mathrm{O} 7-\delta$ coated conductor cables for electric power transmission and magnet applications", Supercond. Sci. Technol., vol. 24, 2011, 042001-4.

[4] R. Champailler, N. Yanagi, G. Bansal, H. Tamura, T. Mito, S. Imagawa, and J. Duchateau, "Experiments of Bending Strain on Reduced-Scale HTS Conductors for Fusion Energy Reactors", IEEE Trans. Appl. Supercond., vol.20, 2010, 1565-8.

[5] C.E. Bruzek et al., "New HTS 2G Round Wires" IEEE Trans. Appl. Supercond., vol. 22, 2012, 5800204.

[6] M. Takayasu, L. Chiesa, L. Bromberg, and J. V. Minervini, "Cabling Method for High Current Conductors Made of HTS Tapes", IEEE Trans. Appl. Supercond., vol. 21, 2011, 2340-3.

[7] D. C. van der Laan and J. W. Ekin, "Dependence of the critical current of $\mathrm{YBa} 2 \mathrm{Cu} 3 \mathrm{O} 7-\delta$ coated conductors on in-plane bending", Supercond. Sci. Technol., vol. 21, 2008, 115002-8. 